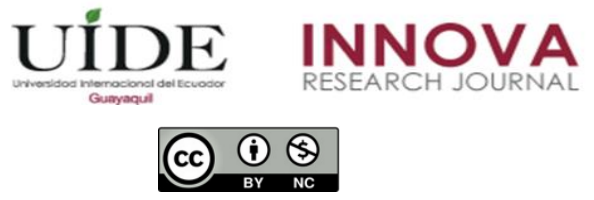

INNOVA Research Journal, ISSN 2477-9024

(Mayo-Agosto 2020). Vol. 5, No.2 pp. 113-132

DOI: https://doi.org/10.33890/innova.v5.n2.2020.1274

URL: http://revistas.uide.edu.ec/index.php/innova/index

Correo: innova@uide.edu.ec

\title{
Las certificaciones como estrategia para la competitividad de las empresas exportadoras
}

\section{Certifications as a strategy for the competitiveness of exporting companies}

Darianny Estefanía Mora-Córdova

https://orcid.org/0000-0001-5248-5167

Arleth Abigail Lituma-Loja

https://orcid.org/0000-0002-3932-1202

Mayiya Lisbeth González-Illescas

http://orcid.org/0000-0002-5219-3807

Universidad Técnica de Machala, Ecuador

Autor por correspondencia: demora_est@utmachala.edu.ec; alituma_est@utmachala.edu.ec; mlgonzalez@utmachala.edu.ec

Fecha de recepción: 17 de diciembre de 2019 - Fecha de aceptación: 23 de marzo de 2020

\section{Resumen}

Las continuas transformaciones de las condiciones de los mercados internacionales representan para las empresas un desafío permanente, poniendo a prueba su capacidad de respuesta y adaptación a los nuevos escenarios. Ante la premisa de cumplir con las exigentes normativas y brindar confianza a los consumidores, las empresas exportadoras han adoptado las certificaciones de distinta índole, como estrategia para defender su sostenibilidad en mercados de cambios acelerados. Este trabajo se plantea como objetivo analizar las certificaciones de mayor contribución a la competitividad de las empresas exportadoras ecuatorianas. El análisis se enfoca en cinco productos del sector agropecuario. Se ha utilizado el método teórico analítico-sintético, además de la revisión bibliográfica de artículos de revistas científicas y documentos de organismos oficiales. Entre los principales resultados se destaca que las certificaciones más utilizadas son aquellas relacionadas al ámbito de la calidad, la seguridad alimentaria, la producción orgánica y la responsabilidad social, como forma de asegurar sus procesos y proyectar valor en los mercados altamente competitivos.

Palabras claves: certificaciones; estrategia de diferenciación; competitividad; empresas exportadoras; sector primario

\begin{abstract}
The continuous transformations of the conditions of the international markets represent a permanent challenge for the companies, testing their capacity to respond and the adaptation to the new scenarios. In face of the premise of complying with the demanding normative and provide confidence to consumers, the exporting companies have adopted certifications of different nature, as a strategy to defend their sustainability in fast-changing markets. This labor aims to analyze the certifications with the greatest contribution to the competitiveness of Ecuadorian exporting companies. The analysis focuses on five products of the agricultural sector. The analytical-
\end{abstract}


synthetic theoretical method has been used, in addition of the bibliographic review of articles of scientific journals and documents of official organizations. Among the main results it stands out that the most used certifications are those related to the ambit of quality, food security, organic production and social responsibility, as a way of ensuring their processes and project value in highly competitive markets.

Key words: certifications; differentiation strategy; competitiveness; export companies; primary sector

\section{Introducción}

Los mercados internacionales se caracterizan por cambios continuos, situación que las empresas exportadoras pueden percibir como oportunidades y amenazas para su expansión. El desarrollo de capacidades para adaptarse a las nuevas condiciones, en paralelo a la generación y mantenimiento de ventajas competitivas se convierten en un reto permanente que las empresas deben enfrentar.

La competitividad en los mercados de productos alimenticios, supera la situación de rivalidad entre los participantes, los cuales intentan definir estrategias para mejorar su oferta y alcanzar sus objetivos de crecimiento. Llamas, Del Carmen y Moreno (2018) explican que el cumplimiento de las estrictas normativas sanitarias y la necesidad de brindar confianza a los consumidores, se identifican como factores relevantes para la sostenibilidad empresarial, requiriendo un ajuste de acciones que permitan afianzar la relación con los actores del comercio internacional.

La respuesta proactiva de las empresas para enfrentar los temas relacionados a la demanda de calidad, seguridad y protección ambiental, entre otros, se identifica en el carácter voluntario de las exportadoras al adoptar medidas que van más allá de lo exigido por las normativas de cumplimiento obligatorio que establecen los marcos regulatorios para la entrada de productos.

En este contexto, las certificaciones cumplen un rol importante en la generación de valor estratégico para las empresas, toda vez que robustecen su reputación y compromiso con la sociedad. La implementación de procesos que conducen a la obtención de certificaciones, como estrategia empresarial, favorece las oportunidades para dar a conocer las prácticas que se siguen para la exportación de productos, aumentando las posibilidades de ingresar a nuevos mercados.

Las empresas ecuatorianas que participan con su oferta exportable en la economía global, evidencian un esfuerzo por defender su competitividad, al incorporar certificaciones que revelan su compromiso con la adopción voluntaria de estándares internacionales.

Al hilo de lo expuesto, este trabajo se guía por la interrogante de definir ¿cuáles son las certificaciones que generan mayor ventaja competitiva a las empresas ecuatorianas en los mercados internacionales? Para dar respuesta a la pregunta planteada se revisan los casos de las empresas que destacan por sus resultados de ventas, asociadas a cinco productos del sector exportador agropecuario. En este sentido, se propone el objetivo de analizar las certificaciones de mayor contribución a la competitividad de las empresas exportadoras ecuatorianas. 
La investigación se desarrolló bajo un enfoque cualitativo, mediante la revisión de artículos de revistas científicas y documentos de organismos oficiales. Se seleccionaron tres empresas de cinco sectores agropecuarios para su análisis. La metodología empleada corresponde al método teórico analítico-sintético.

La principal contribución de este trabajo se circunscribe en la identificación de los tipos de certificación que han adoptado las empresas ubicadas en el ranking de exportación, destacando en el análisis la capacidad de gestión del conocimiento y las habilidades para adaptarse al dinamismo de los mercados (Fayos, Calderón y Frasquet, 2017). El reconocimiento de las prácticas empresariales que priorizan la repercusión de las certificaciones, podría constituirse en un referente para la toma de decisiones de los empresarios involucrados en los sectores analizados.

La estructura del trabajo comprende la introducción donde se presentan los antecedentes y objetivos de la investigación, seguido de la revisión de literatura que cubre los constructos de estrategia y competitividad. Se abordan las certificaciones desde una perspectiva de estrategia de diferenciación, identificando los tipos de certificaciones internacionales de mayor uso y difusión en el intercambio comercial. Luego de la descripción de la metodología se presentan los resultados enfocados por producto agropecuario y de acuerdo a las empresas que sobresalen por sus resultados. Las conclusiones se presentan a manera de consideraciones que asocian el dinamismo del mercado y el impacto de las certificaciones. Finalmente se exponen las limitaciones del estudio y las líneas de investigación que se podrían abordar a futuro.

\section{Revisión de la literatura}

\section{Estrategias para la competitividad de las empresas}

Como punto de referencia teórico de amplia aceptación, la estrategia, según Chandler (1962), comprende la consecución de objetivos, considerando la asignación de recursos a largo plazo. Andrews (1971), también considera la planificación como parte fundamental de la estrategia, para conducir a la empresa a conseguir sus objetivos. En una clara evolución de las perspectivas, Ohmae (1989) describe a la estrategia como el conjunto de acciones empleadas para potenciar los factores diferenciadores de la competencia.

La estrategia en la empresa es un soporte importante para su desarrollo. Es un elemento fundamental para la proyección de la internacionalización (Heiss, 2017). La implementación de estrategias favorece la adaptación de las empresas a los cambios que se desarrollan en los mercados. Para la explicación de la estrategia, los autores adoptan diversos enfoques, no obstante, coinciden en señalar que los beneficios de su aplicación se pueden visibilizar en la innovación y en la generación de valor agregado de los procesos organizacionales.

Para incorporar al análisis una perspectiva contemporánea, en la tabla 1 se recogen las propuestas de varios autores sobre la estrategia. 


\section{Tabla 1}

Conceptualización de estrategias

\begin{tabular}{cl}
\hline Autor & \multicolumn{1}{c}{ Descripción } \\
\hline Villa, (2016) & $\begin{array}{l}\text { Las estrategias a nivel organizacional, producen un fuerte impacto si son } \\
\text { adecuadas en el momento necesario, garantizando la efectividad del desarrollo } \\
\text { y promoviendo la internacionalización y mejorando las circunstancias de la } \\
\text { empresa de manera positiva. }\end{array}$ \\
\hline Bojórquez y Palazuelo, & $\begin{array}{l}\text { Las estrategias se caracterizan por brindar a las empresas la oportunidad de } \\
\text { alcanzar las metas y objetivos, acoplando los recursos y medios para la } \\
\text { aproximación de resultados positivos, ayudando a distribuir los recursos de } \\
\text { manera viable y vinculadas a sus capacidades internas, otorgando una } \\
\text { perspectiva de la actividad de los mercados y el cambio de los competidores. }\end{array}$ \\
\hline & $\begin{array}{l}\text { Las estrategias deben ser direccionadas como procesos que permitan a las } \\
\text { empresas afianzar la competitividad, promover los conocimientos de la } \\
\text { innovación para garantizar la fortaleza a través de la implementación de los } \\
\text { modelos de negocio actuales. }\end{array}$ \\
\hline & $\begin{array}{l}\text { Las estrategias requieren de la evaluación de las fortalezas de la organización, } \\
\text { factores internos y externos, como los requerimientos gubernamentales y } \\
\text { factores políticos. Una adecuada valoración de estos elementos favorecerá la } \\
\text { definición de actividades que permitan lograr los objetivos empresariales. }\end{array}$ \\
\hline
\end{tabular}

Fuente: Elaboración propia a partir de autores

La implementación de las estrategias puede contribuir a mejorar la competitividad de las organizaciones (Huerta, Sandoval y Preciado, 2016). En el contexto de mercados globalizados, la competencia entre las empresas se intensifica, de tal manera que la proactividad se destaca como una capacidad indispensable para identificar los cambios y adaptarse a ellos.

La relación entre la estrategia y la competitividad se puede percibir en las iniciativas planificadas a largo plazo, las que adopta la empresa para anticiparse a los competidores. Así, la competitividad se relaciona con los resultados de las empresas, los cuales pueden ser visibilizados en un mejor posicionamiento en el mercado, mayores ventas, en definitiva, en un conjunto de factores que hacen que los productos servicios sean elegibles a diferencia de la propuesta de oferta de la competencia.

De acuerdo a Arboleda, Sánchez y Sanabria (2016), la competitividad tiene una relación estrecha con la innovación, a partir de la cual se pueden modificar las estructuras organizacionales para lograr productos y servicios adaptados a la demanda cambiante de los mercados. Con este planteamiento coinciden Larios-Gómez, Cuevas-Vargas y Estrada-Rodríguez (2016), quienes relacionan la competitividad con la capacidad de adaptación a los nuevas condiciones del mercado.

Siguiendo a Ibarra, González y Demuner (2017), la competitividad puede ser medida por el crecimiento de la producción y la capacidad que tienen las empresas para incrementar su participación en mercados internacionales mediante exportaciones o inversiones extranjeras.

Con lo expuesto se puede sostener que las empresas para ser más competitivas pueden adoptar estrategias que les permitan hacer perceptibles los factores de distinción entre sus 
competidores. Así, la diferenciación a partir de modelos de gestión, acordes a estándares internacionales, es una alternativa que están utilizando las empresas de éxito en los mercados.

\section{Las certificaciones como estrategia de diferenciación}

Las certificaciones son empleadas en el comercio internacional para garantizar el cumplimiento de las normativas que establece el mercado en general y los organismos reguladores en particular (Ceballos, 2016). A través de las certificaciones las empresas proyectan su compromiso con la seguridad de los consumidores y los estándares del mercado en distintos ámbitos (Vásquez-Tejos y Torres-Vallejos, 2018).

La certificación se considera como una estrategia para robustecer la reputación de las empresas, vinculada al cumplimiento de estrictos protocolos sobre los procesos de producción. Siguiendo a Chirinos y Rosado (2016) y Méndez (2017), la certificación se identifica como una estrategia de diferenciación que las empresas implementan para generar y mantener su posición competitiva.

En la tabla 2 se recogen los puntos de vista de diferentes autores sobre los aspectos que comprenden las certificaciones, así como sus beneficios.

\section{Tabla 2}

Conceptualización de certificación

\begin{tabular}{cl}
\hline Autor & \multicolumn{1}{c}{ Descripción } \\
\hline $\begin{array}{c}\text { Fonseca, Muñóz y Cleves, } \\
\text { (2011) }\end{array}$ & $\begin{array}{l}\text { La certificación es una corroboración que otorgan ciertos organismos } \\
\text { especializados de modo formal y escrito para los productores para justificar de } \\
\text { que cumplen con las normas o alguna otra especificación que quieran ofrecer. }\end{array}$ \\
\hline $\begin{array}{c}\text { Duque, Cardona, y } \\
\text { Rendón, (2013) }\end{array}$ & $\begin{array}{l}\text { Una certificación es de carácter organizacional ya que incluye las políticas y los } \\
\text { objetivos establecidos, afianzando la sostenibilidad y el cumplimiento de los } \\
\text { estándares de calidad en los procesos, sistema, competencias y la mejora } \\
\text { continua, influenciando a lograr un nivel óptimo de competitividad. }\end{array}$ \\
\hline Ceballos, (2016) & $\begin{array}{l}\text { Las certificaciones internacionales, son aquella opción que emplean las empresas } \\
\text { con el fin de optimizar su productividad, llegar a mercados mayormente } \\
\text { atractivos y lograr establecer confianza en los mismos. }\end{array}$ \\
\hline Rangel, Arredondo y yejel, (2017) & $\begin{array}{l}\text { Las certificaciones incluyen un conjunto de tareas que califican a un producto y } \\
\text { que requieren de ciertos requisitos. Para lograrlo, se necesita un organismo que } \\
\text { determine los pasos a seguir para alcanzar la certificación deseada y así tener la } \\
\text { garantía de forma escrita que los productos cumplen todas las normas, brindando } \\
\text { a los productores beneficios y a su vez al consumidor ofrecerle calidad. }\end{array}$ \\
\hline $\begin{array}{l}\text { Las certificaciones tanto en productos como en procesos mejoran o aumentan la } \\
\text { posibilidad de ingreso a un mercado internacional, llegando a beneficiar a los } \\
\text { productores con mejores precios. Por otro lado, las empresas buscan } \\
\text { certificadoras eficientes que garanticen la calidad para los compradores y que las } \\
\text { normas que requieran los países a donde pretende llegar el producto sean } \\
\text { cumplidas. }\end{array}$ \\
\hline Funtreras-Valenzuela et \\
al., (2018)
\end{tabular}

Fuente: Elaboración propia a partir de los autores citados

Después de la revisión expuesta, se puede interpretar que las certificaciones equivalen a una constancia formal, que se obtiene a través del cumplimiento de procedimientos acorde a las exigencias específicas, o ámbito de la certificación. Contribuyen a justificar el cumplimiento de 
normativas que garantizan calidad ya sea de un producto, servicio o proceso, en el cual se ve beneficiado el consumidor y la empresa.

Existen dos tipos de certificaciones: voluntarias y obligatorias. En las primeras, las empresas pueden optar por obtenerlas para generar un nivel de confianza más alto para los compradores y las otras en cambio son exigidas ya sea para lograr la exportación de un producto o cumplir con algún proceso, respetando las normativas exigidas internacionalmente. En tabla 3 se amplían las diferencias entre las certificaciones voluntarias y obligatorias.

\section{Tabla 3}

Tipos de certificaciones

\begin{tabular}{ll}
\hline Certificaciones voluntarias & \multicolumn{1}{c}{ Certificaciones Obligatorias } \\
\hline Son aquellas que el vendedor obtiene de forma & Son aquellas que están implantadas como requisito o \\
voluntaria para brindar a sus clientes seguridad, disposición en cada uno de los países para proceder a \\
confianza y muchas veces demostrar el cuidado al & la compra o venta de productos. Estas evidencian que \\
medio ambiente. Además, estas certificaciones & las mercancías que ingresan o salen de un país \\
pueden ser exigidas por el comprador, de esta forma & cumplen con todas las normas que exigen los \\
se garantiza que para la obtención del producto se & mercados. \\
actuó de manera responsable en el ámbito económico, & \\
social y ambiental.
\end{tabular}

Fuente: Adaptado a partir de Grupo Bancolombia (2019) y Procolombia (2018).

Existen distintas certificaciones internacionales, ya sea para productos, servicios, procesos, de responsabilidad social empresarial, para alimentos, entre otros. Para la presente investigación se ha seleccionado 23 de las más utilizadas organizadas en la tabla 5.

En la tabla 4 abordamos la certificación desde distintos campos, incorporando la descripción de las prácticas organizacionales que marcarían la diferenciación entre las empresas que adoptan las certificaciones como estrategia para proyectar valor agregado a sus clientes.

\section{Tabla 4}

Certificaciones internacionales más utilizadas

\begin{tabular}{cl}
\hline Certificación & \multicolumn{1}{c}{ Descripción } \\
\hline & $\begin{array}{l}\text { Contribuye a la identificación de los riesgos y las oportunidades, garantizando la mejora de la } \\
\text { producción y la calidad de los bienes (Chen, Anchecta, Lee y Dahlgaard, 2016). Todas las } \\
\text { empresas que estén certificadas bajo un Sistema de Gestión de Calidad dentro de esta norma, } \\
\text { serán altamente competitivas, facilitando el ingreso a los nuevos mercados, otorgando valor } \\
\text { agregado a cada producto y mejorando la capacidad productiva (Castro y Rodríguez, 2017; } \\
\text { Fonseca y Domingues, 2017). }\end{array}$ \\
\hline & $\begin{array}{l}\text { Bajo el Sistema de Gestión Ambiental se acogen modelos innovadores que minimizan los } \\
\text { impactos ambientales, fomenta las prácticas de prevención de la contaminación (Acuña, }\end{array}$ \\
Figueroa y Wilches, 2017). La implementación de un SGA en las empresas incluye beneficios \\
como la reducción de costos, bajar los precios, el acercamiento a nuevos mercados, mejorar la \\
relación con las empresas competidoras, y brinda mayor estatus a la empresa en los mercados \\
(Alaña, Gonzaga y González, 2017).
\end{tabular}

La ISO 22000 requiere que las empresas cumplan con las condiciones fundamentales de

ISO 22000 ambiente de trabajo para la producción, manipulación y suministro en la cadena alimentaria, para que el producto final sea de mayor calidad para el consumo humano (Navarro, Pérez y Estrada, 2016). En los últimos años la ISO 22000 se ha posicionado como una certificación 


\begin{tabular}{ll}
\hline Certificación & \multicolumn{1}{c}{ Descripción } \\
\hline & necesaria para los procesos de los alimentos, precisando los requisitos que contrarresten los \\
& peligros, garantizando la calidad e idoneidad de los alimentos, afianzando la seguridad para el \\
& consumo humano y animal (Gil, Ruiz, Escrivá, Font y Manyes, 2017).
\end{tabular}

Permite que las prácticas agrícolas se ejecuten de una forma adecuada y armónica en toda la cadena de valor, con los productos de calidad, reduciendo costos en transacción y fomentando la relación e información entre los productores de las granjas de diferentes países y los Global minoristas, mejorando el comercio internacional (Fiankor, Flachsbarth, Masood y Brümme, G.A.P. 2017). Además, ejecuta operaciones que integra el controlar los diferentes actores y elementos en el proceso de producción, lo cual garantiza una trazabilidad que se adapte a los requerimientos de mercados internacionales, conociendo al producto por medio de la identificación y control de la seguridad alimentaria (Figueredo, Rincón y Salazar, 2018).

Esta certificación se direcciona en los productores y los operadores de comercio justo que como objetivo buscan mejorar las condiciones de vida, la preservación y los procesos de producción certificados bajo estándares equitativos, incluyendo las primas necesarias a precios justos y competitivos en dependencia de los esquemas de esta certificación (Khatri, Ranjan y Grote, 2016). Busca transparencia, y equidad en el comercio internacional, brindando Fairtrade condiciones mejores y garantizando que se respeten los derechos de los pequeños productores y trabajadores. En el caso de aquellas empresas que poseen un gran número de mano de obra, esta certificación compromete al compromiso de la no discriminación, respeto del salario establecido, regulación de número de horas de trabajo, entre otros factores que aseguren beneficios para los trabajadores (Staricco y Naranjo, 2017).

\begin{tabular}{|c|c|}
\hline $\begin{array}{c}\text { Best } \\
\text { Aquaculture } \\
\text { practices } \\
\text { (BAP) }\end{array}$ & $\begin{array}{l}\text { Ciertas prácticas de acuicultura, han provocado un impacto en el medio ambiente, el hábitat } \\
\text { natural de peces ha sido el que mayormente se ha visto afectado (Mehady y Rumana, 2017). } \\
\text { Las empresas que adopten una certificación BAP demostraran a los mercados que mejoran los } \\
\text { estándares ambientales por medio de la aplicación de prácticas sostenibles, dando un resultado } \\
\text { significativo al producto final (Soley, Wuyang y Vassalos, 2019). }\end{array}$ \\
\hline $\begin{array}{l}\text { Business } \\
\text { Alliance for } \\
\text { Secure } \\
\text { Commerce } \\
\text { (BASC) }\end{array}$ & $\begin{array}{l}\text { La certificación BASC tiene como propósito el garantizar efectividad y eficiencia en las } \\
\text { operaciones, se planean condiciones que promueven el mejoramiento de los procesos } \\
\text { logísticos, plantas y equipo de las organizaciones (Fontalvo y DeLaHoz, 2018). La } \\
\text { certificación BASC, disminuye los riesgos de exposición de las mercancías, es decir, tiene un } \\
\text { valor indispensable para las empresas que se dedican a la transportación interna de mercancías, } \\
\text { reduciendo los problemas de robo, contaminación ilícita y contrabando (Veliz y Díaz, 2019). }\end{array}$ \\
\hline Dolpl & $\begin{array}{l}\text { La adaptación de esta certificación permite a las empresas ejercer mejores prácticas pesqueras } \\
\text { y proyecta a que se provoque una reducción a los impactos negativos hacia los delfines ya que } \\
\text { son los que mayormente se ven afectados en la pesca de atún, dicha certificación aporta un } \\
\text { efecto positivo en las decisiones de mercado y la producción de terceros (Baroncini y Brunel, } \\
\text { 2019). }\end{array}$ \\
\hline $\begin{array}{l}\text { Occupational } \\
\text { Health \& } \\
\text { Safety } \\
\text { Advisory } \\
\text { Services: } \\
18001 \\
\text { (OHSAS) }\end{array}$ & $\begin{array}{l}\text { La certificación OHSAS } 18001 \text { garantiza que las organizaciones apliquen en su sistema una } \\
\text { gestión de salud y seguridad entre los actores para lograr el control de los riesgos y alcanzar el } \\
\text { fortalecimiento de las relaciones internas y externas de una empresa incrementando el poder } \\
\text { de la negociación entre accionistas, clientes, el gobierno, aseguradores y sindicatos (Riaño- } \\
\text { Casallas, Hoyos y Pacheco, 2016). Toda organización que implemente una certificación } \\
\text { OHSAS deberá adoptar lo que la norma determina para su mejora continua (Bedoya, } \\
\text { Severiche, Sierra y Ferreira, 2017). }\end{array}$ \\
\hline
\end{tabular}

British Retail

Consortium - La inocuidad alimentaria establece un chequeo en el rendimiento y los distintos procesos de

Food Safety los alimentos apoyándose en desempeñar pruebas al producto final que efectúen una

Standard inspección regular (Jadi, 2016).

(BRC) 


\begin{tabular}{cl}
\hline Certificación & \multicolumn{1}{c}{ Descripción } \\
& $\begin{array}{l}\text { Proporciona a los productos marinos una identificación que permite a los consumidores } \\
\text { conocer bajo esta etiqueta que las prácticas pesqueras tienen un proceso de protección }\end{array}$ \\
& ecológica. Esta certificación garantiza la sostenibilidad, disminución de la sobrepesca, \\
Marine & mantenimiento de los ecosistemas acuáticos y minimizar los efectos ambientales (Lajus, \\
Stewardship & Stogova y Keskitalo, 2018). MSC elimina las malas prácticas de pesca y fomenta el \\
Council & conocimiento de las políticas que generan efectos negativos en la industria para su acceso a \\
(MSC) & los mercados internacionales (Kohne, 2019). Es considerada como un estándar de protección \\
& en términos de pesca y su adaptación en una organización puede generar ventaja competitiva \\
& en mercados como Norteamérica y el noroeste de Europa (Wijen y Chiroleu-Assouline, 2019).
\end{tabular}

Este tipo de certificación actúa como un instrumento que mejora la calidad y reduce los costos, siendo extensamente aprobado por los minoristas de Alemania y Francia, incluyendo las

IFS Food marcas de los fabricantes, provocando que se reemplace a la Organización Internacional de Normalización de las industrias alimentarias europeas (Fontaine, Raposo, Millán, Sanjuán y Carrascosa, 2018).

La certificación USDA Organic ha tenido un impacto positive en EE. UU ya que los consumidores han adoptado un nivel de conciencia y respuesta positiva dentro de lo que engloba esta herramienta, ya que presentan conformidad a diferencia de otras etiquetas (Zhang

USDA y Boldt, 2017). La certificación USDA Organic incluye la participación de agentes los, cuales

Organic se encargan de que las organizaciones solicitantes de dicho certificado, cumplan con diferentes especificaciones importantes como cultivo, proceso de los productos, ganado y cultivos silvestres (Olsen, DeCarlo y Passarelli, 2017).

\begin{tabular}{ll}
\hline UTZ, como certificación mejora los procesos de cultivo y producción en un 20\% ya que \\
garantiza la reducción y buen manejo de los recursos a diferencia de las granjas no certificadas \\
bajo este estándar (Haggar, Soto, Casanoves y De Melo, 2017). Ofrece grandes beneficios para \\
GTZ Kapeh \\
que los solicitantes se sientan motivados, incorpora el precio de la prima del café certificado, \\
precios transparentes, fortalece la negociación de los productores, asistencia para la \\
producción en cuestiones de calidad y obtención del respaldo de organismos no no \\
gubernamentales para lograr certificarse (Latynskiy y Berger, 2017).
\end{tabular}

\begin{tabular}{cl}
\hline $\begin{array}{c}\text { Japanese } \\
\text { Agricultural } \\
\text { Standars } \\
\text { (JAS) }\end{array}$ & $\begin{array}{l}\text { Esta certificación es impuesta para todos los productos alimenticios, a pesar de que los mismos } \\
\text { ya cuenten con algún certificado orgánico de otro país. Todo esto es con el fin de garantizar } \\
\text { calidad al consumidor japonés y asegurarle de que hizo una buena elección (Gracia, 2018). }\end{array}$ \\
\hline & $\begin{array}{l}\text { Kosher, es aquella certificación que establece el control de la calidad alimenticia conforme a } \\
\text { las normativas hebreas. Al certificarse con Kosher, los productos respetan las normas bíblicas }\end{array}$ \\
La Unión & $\begin{array}{l}\text { de la ley judía. Por ejemplo, la carne, garantiza a los consumidores que ésta ha sido sacrificada } \\
\text { de acuerdo a las disposiciones de la religión certificándola para la confesión judía (Liñán },\end{array}$ \\
Ortodoxa \\
Kosher & $\begin{array}{l}\text { 2017). La etiqueta Kosher, asegura confianza cuando se trata de calidad y garantiza de que son } \\
\text { productos limpios o puros, siendo aptos para el consumo de los judíos (López, 2019). }\end{array}$
\end{tabular}

Esta certificación es de gran credibilidad y confianza sobre los productos alimenticios para los consumidores, es decir que estos no contengan en su composición nada que sea considerado

Halal ilícito (Wibowo y Ahmad, 2016). Dentro de la ley islámica, para la población musulmana es de gran importancia que los alimentos cuenten con todos los permisos para ser consumidos, debido a que consideran que esto impulsa el bienestar y el comportamiento de las personas (Krishnan, Omar, Zahran, Syazwan y Alyaa, 2017). 


\begin{tabular}{|c|c|}
\hline Certificación & Descripción \\
\hline $\begin{array}{l}\text { Buenas } \\
\text { prácticas } \\
\text { agrícolas } \\
\text { (BPA) }\end{array}$ & $\begin{array}{l}\text { Esta certificación es una importante herramienta para la sustentabilidad ambiental y social de } \\
\text { los productos agrícolas, garantizando ser sanos e inofensivos para el consumo, realizando } \\
\text { procesos productivos en donde se realice el uso razonable de los recursos. Asimismo, las BPA } \\
\text { ayudan que los productores sean competitivos, ofreciendo un producto de calidad a un mejor } \\
\text { precio y los mismos puedan acceder a mercados nuevos. Además, al implementar las BPA, se } \\
\text { protege el ambiente, se aporta al cuidado de la salud humana y mejora la calidad de vida (Rizo- } \\
\text { Mustelier, Vuelta-Lorenzo y Lorenzo-García, 2017). }\end{array}$ \\
\hline $\begin{array}{l}\text { Buenas } \\
\text { prácticas de } \\
\text { manufactura } \\
(\mathrm{BPM})\end{array}$ & $\begin{array}{l}\text { Es un método de control preventivo que involucra la higiene, preparación, empaque y } \\
\text { almacenaje de alimentos, para de esta forma mantener las condiciones sanitarias adecuadas y } \\
\text { evitar la contaminación de los mismos (Fierro, Duque y Pérez, 2017). Esta certificación mejora } \\
\text { las condiciones de los productos alimenticios para los consumidores, principalmente los } \\
\text { productores obtienen mayores beneficios en los mercados (Gonzaga, Morán y Brito, 2017). }\end{array}$ \\
\hline $\begin{array}{l}\text { Sistema de } \\
\text { análisis de } \\
\text { peligros y } \\
\text { puntos } \\
\text { críticos de } \\
\text { control } \\
\text { (HACCP) }\end{array}$ & $\begin{array}{l}\text { Es empleada en las industrias que se dedican a producir alimentos y en aquellas que se dedican } \\
\text { a producir insumos que mantengan contacto directo con alimentos. A esta certificación se la } \\
\text { ha identificado como el mejor método que asegura la inocuidad alimentaria, logrando que los } \\
\text { distintos países adopten esta norma como un principal requisito en este tipo de industria, } \\
\text { asegurando calidad en cada alimento para el consumo humano. Además, es importante } \\
\text { destacar que también HACCP es aplicada en la industria farmacéutica (Procolombia, 2018). }\end{array}$ \\
\hline Rainforest & $\begin{array}{l}\text { Rainforest Alliance Certified es una certificación que garantiza a los consumidores que los } \\
\text { productos fueron cultivados y cosechados cumpliendo cada una de las normas y usando } \\
\text { prácticas ambiental y socialmente responsables (Vera y Cañón, 2018). }\end{array}$ \\
\hline Veriflora & $\begin{array}{l}\text { Esta certificación es parte de un plan de etiqueta ecológica y agricultura sustentable, siendo } \\
\text { reconocida en las industrias de floricultura y de horticultura. Aquellos productores, } \\
\text { distribuidores, florerías al por mayor y menor o cualquier empresa relacionada con venta de } \\
\text { flores y plantas que cuenten con la certificación Veriflora, se comprometen a proporcionar } \\
\text { calidad, debido a que tienen una producción con normas rigurosas, con responsabilidad } \\
\text { ambiental, y cuida de la salud de trabajadores y la comunidad en general (Proecuador, 2013). }\end{array}$ \\
\hline
\end{tabular}

Fuente: Elaboración propia

Del conjunto de certificaciones citadas, se puede deducir que cubren varios campos de gestión. Siendo de interés para las empresas, la implementación de protocolos y su certificación, contribuye a mantener el control de los procesos de producción, la salud organizacional, el cuidado del medio ambiente, entre otros.

Existen ciertas certificaciones que al ser implementadas favorecen el ingreso a nuevos mercados, ya que ofrecen calidad a los compradores de otras culturas, brindándoles seguridad en lo que van a consumir.

\section{Metodología}

Este trabajo es desarrollado con un enfoque cualitativo, de acuerdo al objetivo planteado de analizar las certificaciones de mayor contribución a la competitividad de las empresas exportadoras ecuatorianas.

Se ha empleado el método teórico analítico-sintético, considerado un método acorde al propósito de analizar los distintos tipos de certificaciones. Como fuente de información se utilizan artículos de revistas científicas, documentos de organismos oficiales y páginas webs de empresas exportadoras. 
La presente investigación es de carácter descriptivo, para lo cual se ha sistematizado la clasificación de las certificaciones de acuerdo a cinco productos de exportación, pertenecientes al sector agropecuario, esto es, banano, cacao, flores, café y camarón.

La selección de productos se sostiene en la importancia que revisten para la generación de ingresos al país por exportaciones. Respecto a la selección de las empresas, se determinó escoger aquellas con mayor desempeño. Para el producto 1 (tabla 6) se utilizó un cuadro estadístico de las mejores exportadoras hasta julio del 2019 publicado por la Asociación de Exportadores de Banano del Ecuador; para el producto 2, 3 y 4 (tabla 7, 8 y 9 respectivamente) se escogió a las empresas del ranking empresarial 2018 de la Revista Ekos, seleccionando a las mismas según sus ingresos. Para el producto 5 (tabla 10) se eligió a las empresas por exportador según las estadísticas de enero - septiembre del 2019 publicadas por la Asociación Nacional de Exportadores de Café.

Bajo la metodología declarada, en el siguiente apartado abordamos los resultados de la investigación.

\section{Resultados}

En coherencia al objetivo planteado y la interrogante que guía esta investigación, los resultados se obtienen del análisis de los recursos de información definidos anteriormente. En este sentido, pretendemos responder a la pregunta ¿cuáles son las certificaciones que generan mayor ventaja competitiva a las empresas ecuatorianas en los mercados internacionales?

La presentación de los resultados se divide en dos partes. Para la primera parte se consideró la identificación del tipo de certificaciones de mayor uso, en relación al producto agropecuario de exportación. Como segunda parte de los resultados, se tomaron como referencia los rankings de exportación para identificar a las principales empresas que se destacan por sus ventas, luego se indagó en la página web de cada empresa el tipo de certificación que emplean.

El mercado de productos primarios se destaca por la gran cantidad de competidores, quienes representan a una corriente de ofertantes con características de alta diferenciación. De forma paralela, la situación económica de menor crecimiento de los principales países compradores, se refleja en una demanda moderada de productos primarios, dicho comportamiento incrementa la complejidad de las empresas para permanecer en el mercado. Los sectores que han logrado mantenerse con una demanda sostenida, a pesar de las adversidades descritas, sobresalen por sus resultados de exportación, la explicación puede asociarse a la implementación de estrategias como la certificación.

En la tabla 5 se presentan cinco productos del sector primario, los cuales se identifican como los principales productos de exportación no petrolera. En la misma tabla se identifica el tipo de certificación que se relaciona a la selección anterior. 
Tabla 5

Clasificación de certificaciones por sectores productivos

\begin{tabular}{|c|c|c|c|c|c|c|}
\hline & \multirow{3}{*}{ Certificación } & \multicolumn{5}{|c|}{ Sector agropecuario o primario } \\
\hline & & \multicolumn{4}{|c|}{ Sector agrícola } & \multirow{2}{*}{$\begin{array}{c}\begin{array}{c}\text { Sector } \\
\text { pesquero }\end{array} \\
\text { Camarón }\end{array}$} \\
\hline & & Banano & Cacao & Flores & Café & \\
\hline \multirow{4}{*}{ 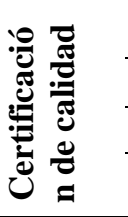 } & ISO 9001 & $\mathrm{x}$ & $\mathrm{x}$ & $\mathrm{x}$ & $\mathrm{X}$ & $\mathrm{X}$ \\
\hline & ISO 14001 & $\mathrm{x}$ & $\mathrm{x}$ & $\mathrm{x}$ & $\mathrm{x}$ & $\mathrm{x}$ \\
\hline & ISO 22000 & $\mathrm{x}$ & $\mathrm{X}$ & $\mathrm{x}$ & $\mathrm{x}$ & $\mathrm{X}$ \\
\hline & OHSAS & $\mathrm{X}$ & $\mathrm{X}$ & $\mathrm{x}$ & $\mathrm{x}$ & $\mathrm{X}$ \\
\hline \multirow{5}{*}{ 苞 } & Global G.A.P. & $\mathrm{x}$ & $\mathrm{x}$ & $\mathrm{x}$ & $\mathrm{x}$ & $\mathrm{x}$ \\
\hline & Veriflora & & & $\mathrm{x}$ & & \\
\hline & Rainforest & $\mathrm{X}$ & $\mathrm{X}$ & $\mathrm{x}$ & $\mathrm{x}$ & \\
\hline & $\begin{array}{l}\text { Buenas prácticas } \\
\text { agrícolas (BPA) }\end{array}$ & $\mathrm{x}$ & $\mathrm{x}$ & $\mathrm{x}$ & $\mathrm{X}$ & \\
\hline & UTZ Kapeh & & & & $\mathrm{x}$ & \\
\hline \multirow{3}{*}{ שֶ } & $\begin{array}{l}\text { Best Aquaculture } \\
\text { practices }\end{array}$ & & & & & $\mathrm{x}$ \\
\hline & Dolphin safe & & & & & $\mathrm{x}$ \\
\hline & $\begin{array}{c}\text { Marine Stewardship } \\
\text { Council }\end{array}$ & & & & & $\mathrm{x}$ \\
\hline \multirow{6}{*}{ 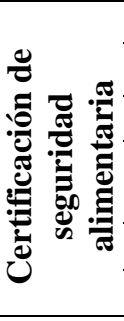 } & BRC & $\mathrm{X}$ & $\mathrm{X}$ & & $\mathrm{x}$ & $\mathrm{X}$ \\
\hline & IFS Food & $\mathrm{x}$ & $\mathrm{x}$ & & $\mathrm{x}$ & $\mathrm{x}$ \\
\hline & HACCP & $\mathrm{x}$ & $\mathrm{X}$ & $\mathrm{x}$ & $\mathrm{x}$ & $\mathrm{x}$ \\
\hline & $\begin{array}{l}\text { Buenas prácticas de } \\
\text { manufactura (BPM) }\end{array}$ & $\mathrm{x}$ & $\mathrm{x}$ & $\mathrm{x}$ & $\mathrm{X}$ & $\mathrm{X}$ \\
\hline & Kosher & $\mathrm{x}$ & $\mathrm{x}$ & $\mathrm{x}$ & $\mathrm{x}$ & $\mathrm{x}$ \\
\hline & Halal & $\mathrm{X}$ & $\mathrm{X}$ & & $\mathrm{X}$ & $\mathrm{X}$ \\
\hline \multirow{2}{*}{ 苞 } & USDA Organic & $\mathrm{x}$ & $\mathrm{X}$ & $\mathrm{x}$ & $\mathrm{x}$ & \\
\hline & JAS & $\mathrm{x}$ & $\mathrm{x}$ & $\mathrm{x}$ & $\mathrm{x}$ & \\
\hline 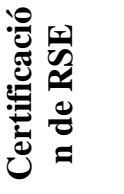 & SA8000 RSE & $\mathrm{x}$ & $\mathrm{X}$ & $\mathrm{x}$ & $\mathrm{x}$ & $\mathrm{x}$ \\
\hline \multirow{2}{*}{ 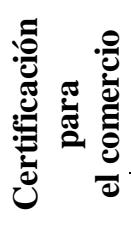 } & Fairtrade & $\mathrm{x}$ & $\mathrm{x}$ & $\mathrm{x}$ & $\mathrm{X}$ & \\
\hline & BASC & $\mathrm{X}$ & $\mathrm{X}$ & $\mathrm{x}$ & $\mathrm{x}$ & $\mathrm{x}$ \\
\hline
\end{tabular}

Fuente: Elaboración propia adaptada de Proecuador (2013). 
Las certificaciones desempeñan un papel importante para las exportaciones ecuatorianas, sin importar el sector al que pertenezcan. Lograr certificarse es un reto, pero brinda grandes beneficios a las empresas, como incrementar sus ventas, fidelizar clientes, conquistar nuevos mercados.

Para la segunda parte de la presentación de los resultados se han elaborado cinco tablas, correspondiendo a los productos: banano, cacao, flores, café y camarón del sector agropecuario o primario. A su vez, se ha seleccionado tres empresas exportadoras para cada producto, las cuales han sido seleccionadas del contraste de fuentes oficiales, en donde se pueda constatar que son aquellas que sobresalen por su desempeño, es decir, volumen de exportación y ventas.

Para el banano se emplearon las estadísticas publicadas por la Asociación de Exportadores de Banano del Ecuador (mejores exportadoras), para el cacao, camarón y flores se consideró a las empresas del ranking empresarial 2018 de la Revista Ekos (según sus ingresos), y para el producto café se seleccionó según estadísticas de la Asociación Nacional de Exportadores de Café (por exportador).

Una vez seleccionadas las tres empresas por producto, se realizó la búsqueda de las certificaciones que poseen cada una, las mismas que fueron extraídas de sus páginas web oficiales.

\section{Banano}

En la tabla 6 se presentan las certificaciones que mantienen las tres principales exportadoras de banano.

\section{Tabla 6}

Producto 1: Banano

\begin{tabular}{lll}
\hline \multicolumn{1}{c}{ Ubesa } & \multicolumn{1}{c}{ Reybanpac } & \multicolumn{1}{c}{ Frutadeli } \\
\hline - Globalgap & $\bullet$ Globalgap & $\bullet$ Globalgap \\
- Rainforest & & \\
- Buenas prácticas de manufacturas & & \\
\hline
\end{tabular}

Fuente: Elaboración propia

La iniciativa de adoptar estándares internacionales es una respuesta a la exigencia por parte de los consumidores, quienes al momento de seleccionar la fruta reconocen y aprecian los procesos que aseguran la calidad y la seguridad.

Es decir, a los consumidores no les importa pagar un poco más por su compra, sino que requieren que estas certificaciones garanticen la inocuidad del producto, respeto al ecosistema, que se realice un comercio justo, que se desarrollen las mejores prácticas agrícolas, en fin, exigen un banano certificado que afiance la calidad y la seguridad.

Todo esto es porque en los mercados internacionales los consumidores si se preocupan por conocer si la fruta que van a consumir se cosecha sin perjudicar el ecosistema, si el trabajador que plantó a la misma tuvo un pago justo y si el proceso de producción se realizó con los mejores estándares. 
El banano es el principal producto de exportación por volumen, medido en toneladas métricas. Mediante una demanda constante, los mercados internacionales reconocen la calidad del banano ecuatoriano y sus certificaciones.

\section{Cacao}

El cacao, en el año 2018 ocupó el segundo lugar como producto de exportación no petrolero (Anecacao, 2018). En los diferentes mercados objetivos existe un alto nivel competitivo liderado por los principales países exportadores Costa de Marfil, Ghana, Indonesia y Ecuador, los cuales que cubren el $80 \%$ de la oferta mundial.

En la tabla 7 se describen las certificaciones con las que operan las principales empresas exportadoras de cacao.

\section{Tabla 7}

Producto 2: Cacao

\begin{tabular}{|c|c|c|}
\hline Ecuador cocoa \& coffee & $\begin{array}{c}\text { Chocolates finos nacionales } \\
\text { COFINA }\end{array}$ & $\begin{array}{c}\text { Cacaos finos ecuatorianos } \\
\text { CAFIESA }\end{array}$ \\
\hline - HACCP & $\begin{array}{l}\text { - Kosher } \\
\text { - FSSC } 22000 \\
\text { - Rainforest } \\
\text { - HACCP } \\
\text { - UTZ Certified Good Inside } \\
\text { - ISO } 9001 \\
\text { - Vegan } \\
\text { - USDA Organic } \\
\text { - Halal Food } \\
\text { - Agricultura ecológica }\end{array}$ & $\begin{array}{l}\text { - FDA } \\
\text { - ISO 22000: } 2005 \\
\text { - Agricultura ecológica } \\
\text { - USDA organic } \\
\text { - Kosher } \\
\text { - HACCP } \\
\text { - } 100 \% \text { Halal Certified }\end{array}$ \\
\hline
\end{tabular}

Las empresas que realizan comercio con el cacao deben adoptar certificaciones que garanticen la calidad de su producto, así como el dar a conocer a los mercados los procesos que realizan, de forma que las certificaciones otorguen información acerca del producto, es decir, si este es orgánico o si cumple con las especificaciones requeridas por mercados como el medio Oriente, por ejemplo.

\section{Flores}

Como producto de exportación, las flores tienen gran acogida por el mercado de Estados Unidos, donde se envían más de 60 variedades, sin embargo, las rosas son las que tienen una mayor demanda. No obstante, existen otros mercados como Canadá Alemania Holanda Italia, Rusia, entre otros, que también importan nuestras flores. En la tabla 8 se describen las certificaciones con las que cuentan las principales exportadoras de flores. 


\section{Tabla 8}

Producto 3: Flores

\begin{tabular}{lll}
\hline \multicolumn{1}{c}{ Esmeralda Farms } & \multicolumn{1}{c}{ Falcon Farms } & Florícola San Isidro Labrador \\
\hline - Flor Ecuador / Certificado & $\bullet$ Rainforest & $\bullet$ BASC \\
Socio-Ambiental & $\bullet$ BASC & $\bullet$ BPM = Flor Ecuador \\
- Rainforest Alliance & $\bullet$ C-TPAT Certified & \\
- BASC & $\bullet$ ISO 14000 & \\
- Eco-Certified / International & $\bullet$ CYD Certified S.A. \\
Silver Rose Award & & \\
\hline
\end{tabular}

Fuente: Elaboración propia

Actualmente, las empresas que exportan flores para mantenerse en los mercados deben incluir distintas certificaciones que indiquen las prácticas agrícolas que desempeñan para conocimiento de los mercados objetivos, además de incluir los sellos que contribuyen a que las empresas cumplan con las normas de sostenibilidad del ecosistema.

\section{Camarón}

En la tabla 5 se expone de manera conjunta a las tres principales exportadoras de camarón junto a las certificaciones con las que complementan su oferta en los mercados internacionales.

Tabla 9

Producto 4: Camarón

\begin{tabular}{|c|c|c|}
\hline Santa Priscila & Omarsa S.A. & $\begin{array}{c}\text { Sociedad Nacional de } \\
\text { Galápagos C.A. - SONGA }\end{array}$ \\
\hline $\begin{array}{l}\text { - Orgánico - Unión Europea } \\
\text { - Orgánico - Ecuador } \\
\text { - Punto Verde } \\
\text { - BRC } 2019 \\
\text { - BPM } \\
\text { - ASC } 2018 \\
\text { - MSC } 2021 \\
\text { - INEN } \\
\text { - Whole Foods Market } 2019 \\
\text { - Ethical Trading Initiative (ETI) } \\
\text { 2017 } \\
\text { - Kosher }\end{array}$ & $\begin{array}{l}\text { - Sustainable Shrimp Partnership } \\
\text { SS } \\
\text { - ASC / Aquaculture Stewardship } \\
\text { Naturland } \\
\text { - BAP - Best Aquaculture } \\
\text { Practices } \\
\text { - Global G.A.P } \\
\text { - BRC } \\
\text { - BASC } \\
\text { - Sedex }\end{array}$ & $\begin{array}{l}\text { - BAP } \\
\text { - ASC Aquaculture Stewardship } \\
\text { Council. } \\
\text { - BRC Global Standard - Food } \\
\text { - HACCP } \\
\text { - BASC, a los estándares de } \\
\text { Seguridad Internacional. } \\
\text { - FSSC 22000, alta certificación } \\
\text { de inocuidad y calidad } \\
\text { alimentaria }\end{array}$ \\
\hline
\end{tabular}

Fuente: Elaboración propia

La mayor cantidad de exportaciones que se realizan del camarón son hacia China, Brasil, EE. UU, Vietnam, entre otros. La fase comercial en los mercados internacionales, conlleva a adquirir las diferentes certificaciones que permiten ingresar a estos mercados, en donde se precisan altos estándares de calidad.

Además, dichas certificaciones permiten que la competencia, el mercado y los consumidores conozcan los sellos y lo que representa el estar sujeto a las normativas, en su mayoría autorizan el conocimiento de las prácticas acuícolas, la inocuidad de los alimentos y la sostenibilidad en las prácticas pesqueras. 


\section{Café}

\section{Tabla 10}

Producto 5: Café

\begin{tabular}{lll}
\hline $\begin{array}{c}\text { Cia Elaborados de café "El } \\
\text { café C.A." }\end{array}$ & \multicolumn{1}{c}{ Cafecom S.A. } & \multicolumn{1}{c}{ Proexports S.A. } \\
\hline$\bullet$ ISO 90001:2008 & $\bullet$ Fairtrade & $\bullet$ UTZ certified \\
$\bullet$ KGC Kosher & $\bullet$ Specialty Coffee Association & $\bullet$ Fairtrade \\
& $\bullet$ Coffe Quality Institute & $\bullet$ Certified Organic OCIA \\
& & $\bullet$ Orgánico Mayacert \\
\hline
\end{tabular}

Fuente: Elaboración propia

El café, siendo uno de los productos más importantes para la economía del Ecuador, ha conseguido introducirse en mercados internacionales por su calidad y las diferentes variedades que ofrece, valorando los aspectos de trazabilidad en cultivo producción, industrialización y comercialización.

Las exportaciones de café por sacos de 60 kilos hasta octubre del 2019 han tenido un total de \$59.915.648,42 (Anecafé, 2019). Los principales mercados del café ecuatoriano en el año 2019 han sido Colombia y Estados Unidos, sin embargo, su mayor cantidad en demanda según el historial que posee es el mercado de europeo.

De modo que la adaptación de las certificaciones es uno de los aspectos más importantes para su participación en los mercados internacionales ya que juegan un papel decisivo en cuanto a las exigencias de los mercados en relación a la inocuidad, las prácticas de manufactura y la calidad del café como producto final, garantizándole seguridad al consumidor.

\section{Conclusiones}

El crecimiento de la concienciación social sobre los efectos de las actividades económicas en el medio ambiente y el compromiso ético de las empresas, ha conducido a una mayor regulación para el acceso a los mercados. En este escenario, las empresas exportadoras se enfrentan a fuertes presiones para mantenerse competitivas. En una actitud proactiva, la adopción de certificaciones se revela como una estrategia para generar competitividad en los mercados internacionales.

Mediante el análisis de cinco productos del sector primario y las empresas más representativas en las exportaciones de dichos productos, se ha identificado el tipo de certificación que utilizan para diferenciarse de la competencia. Entre los principales resultados se destaca que las certificaciones más utilizadas por las exportadoras ecuatorianas son aquellas relacionadas al ámbito de la calidad, la seguridad alimentaria, la producción orgánica y la responsabilidad social, como forma de asegurar sus procesos y proyectar valor en los mercados altamente competitivos.

Los diferentes productos expuestos en los resultados de la presente investigación, son aquellos que mantienen un impacto positivo en la balanza comercial del Ecuador. En paralelo, se revela que las empresas que lideran las exportaciones han adoptado las certificaciones para 
generar confianza al consumidor, garantizando una mayor acogida en los distintos mercados objetivos frente a otras empresas de productos similares.

Los hallazgos son congruentes con las conclusiones de otros autores como Ceballos (2016) y Contreras-Valenzuela et al. (2018), quienes señalan que las certificaciones contribuyen a incrementar las posibilidades de ingresar a nuevos mercados. Reforzando estos argumentos, Vásquez-Tejos y Torres-Vallejos (2018) destacan que las certificaciones garantizan a los consumidores el cumplimiento de los estándares establecidos en el mercado, constituyéndose en una de las principales estrategias para la competitividad, y, por tanto, el mantenimiento en los mercados internacionales.

Finalmente, en función de los objetivos planteados y el enfoque cualitativo aplicado, la contribución de la investigación se hace a partir de datos secundarios, con lo cual se limita la generalización de los resultados a otros sectores. Precisamente esta limitante nos sirve de premisa para esbozar futuras líneas de investigación, en las cuales se plantea ampliar el análisis, considerando la incorporación de muestras paramétricas que permitan medir la percepción de las empresas exportadoras sobre la certificación como estrategia de diferenciación en los mercados internacionales.

\section{Bibliografía}

Acuña, N., Figueroa, L., \& Wilches, M. (2017). Influencia de los sistemas de gestión ambiental ISO 14001 en las organizaciones: caso estudio empresas manufactureras de Barranquilla. Ingeniare. Revista chilena de ingeniería, 25(1), 143-153. doi:http://dx.doi.org/10.4067/S0718-33052017000100143

Alaña, T., Gonzaga, S., \& González, A. (2017). La gestión ambiental en la competitividad de las PYMESdel Ecuador. INNOVA Research Journal, 2(8.1), 236-248. doi:https://doi.org/10.33890/innova.v2.n8.1.2017.371

Andrews, K. R. (1971). The concept of corporate strategy. Homewood, III., Dow Jones-Irwin.

Anecacao. (2018). Sector exportador de cacao. Obtenido de Anecacao: http://www.anecacao.com/index.php/es/estadisticas/estadisticas-actuales.html

Anecafé. (2019). Exportaciones de café 2019. Obtenido de Anecafé: https://www.anecafe.org.ec/exportaciones2019

Arboleda, G. B., Sánchez, A. M., \& Sanabria, N. J. (2016). Factores de éxito competitivo: caso ESAL Barranquilla. Dimensión empresarial, 14(2), 163-179. doi:http://dx.doi.org/10.15665/rde.v14i2.740

Arredondo, F., \& Vázquez, J. (2017). La responsabilidad social de la industria de los videojuegos : una aproximación desde los contenidos. Ciencia y Sociedad, 42(4), 31-39. doi:10.22206/cys.2017.v42i4

Baroncini, E., \& Brunel, C. (2019). A WTO safe harbour for the dolphins: The second compliance proceedings in the US - Tuna II (Mexico) Case. SSRN(10), 1-23.

Bedoya, E., Severiche, C., Sierra, D., \& Ferreira, C. (2017). Integrated management system based on the OHSAS 18001: 2007 and ISO 28000: 2006 Standards for a Logistic Services Company. International Journal of Engineering and Applied Sciences, 4(7), 85-89. 
Bojórquez, M., \& Palazuelos, O. (2017). El comercio electrónico como estrategia de internacionalización de las PYMES. Revista de Investigacion en Tecnologías de la Información, 5(10), 110-115.

Castro, H., \& Rodríguez, F. (2017). Incidencia de la certificación de la norma ISO 9001 en los resultados empresariales. Un caso colombiano. Entre Ciencia e Ingeniería, 11(22), 18-25.

Ceballos, S. L. (julio-diciembre de 2016). Conocimiento y aplicación de las certificaciones internacionales por las empresas del Altiplano del Oriente Antioqueño. Science of human, $1(2), 300-322$.

Chandler, A. D. (1962). Strategy and Structure: Chapters in the history of American Enterprise. Boston: MIT Press.

Chen, C.-k., Anchecta, K., Lee, Y., \& Dahlgaard, J. (2016). A stepwise ISO-based TQM implementation approach using ISO 9001: 2015. Management and Production Engineering Review, 7(4), 65-75. doi:10.1515/mper-2016-0037

Chirinos, C., \& Rosado, J. (2016). Estrategia de diferenciación: el caso de las empresas industriales. Ingeniería Industrial(34), 165-174.

Contreras-Valenzuela, A. C., Preciado-Rodríguez, J. M., Báez-Sañudo, R., Robles-Parra, J. M., Taddel-Bringas, C., \& Valderrain-Benitez, R. A. (2018). Certificaciones agrícolas como conducta estratégica del sistema vid de mesa sonorense. Revista Iberoamericana de Tecnología Postcosecha, 19(1), 4-20.

Duque, Y., Cardona, M., \& Rendón, J. (2013). Responsabilidad Social Empresarial: Teorías, índices, estándares y certificaciones. Cuadernos de Administración, 29(50), 196-206.

Fayos, T., Calderón, H., \& Frasquet, M. (2017). Implantación internacional de los minoristas de moda multicanal: capacidades dinámicas e incrustación en los mercados. Revista de Globalización, Competitividad y Gobernabilidad, $\quad 11(2), \quad 62-79$. doi:https://doi.org/10.3232/GCG.2017.V11.N2.03

Fiankor, D.-D., Flachsbarth, I., Masood, A., \& Brümmer, B. (s.f.). Does globalGAP certification promote agrifood exports? GlobalFood Discussion Papers(112), 1-30.

Fierro, J. P., Duque, F. J., \& Pérez, H. A. (2017). Análisis de la percepción de los consumidores y empleados de una empresa de productos cárnicos de la ciudad de Guayaquil - Ecuador para la aplicación de un sistema de gestión de inocuidad alimentaria. Revista Observatorio de la Economía Latinoamericana.

Figueredo, C., Rincón, N., \& Salazar, N. (2018). Caracterización del cumplimiento inicial de la Norma Global G.A.P., aplicada a pequeños agricultores de Duitama. Entre Ciencia e Ingeniería, 12(23), 32-39. doi:http://dx.doi.org/10.31908/19098367.3700

Fonseca, J. A., Muñóz , N. A., \& Cleves, J. A. (2011). El sistema de gestión de calidad: elemento para la competitividad y la sostenibilidad de la producción agropecuaria colombiana. Revista de investigación agraria y ambiental, 2(1), 9-22.

Fonseca, L., \& Domingues, J. (2017). ISO 9001:2015 Edition-management, quality and value. International Journal for Quality Research, 11(1), 149-158. doi:10.18421/IJQR11.01-09

Fontaine, A., Raposo, A., Millán, R., Sanjuán, E., \& Carrascosa, C. (2018). Degree of implementation and satisfaction in food companies with the International Food Standards (IFS) and British Retail Consortium (BRC) certifications on the Canary Islands. Biomedical and Biopharmaceutical Research, 15(1), 8-26. doi:DOI: 10.19277/bbr.15.1.173 
Fontalvo, T., \& DeLaHoz, E. (2018). Study of financial efficiency in companies certified with the BASC label using Data Envelopment Analysis: Case applied in Cali - Colombia. Entramado, 14(1), 78-87. doi:https://doi.org/10.18041/entramado.2018v14n1.27122

Gil, L., Ruiz, P., Escrivá, L., Font, G., \& Manyes, L. (2017). A decade of food safety management system based on ISO 22000. Revista de toxicología, 34(2), 84-93.

Gonzaga, S., Morán, G. G., \& Brito, B. B. (2017). Análisis exploratorio de buenas prácticas de manufactura del sector camaronero. "Asociación Aprocam JK". Estudio de caso. Universidad y Sociedad, 9(1), 28-35.

Gracia, M. (2018). Comercio México-Japón. Un acercamiento a la exportación mexicana de carne de cerdo. México y la cuenca del pacífico, 7(20), 117-148.

Grupo Bancolombia. (17 de julio de 2019). Grupo Bancolombia. Obtenido de https://www.grupobancolombia.com/wps/portal/negocios-pymes/actualizate/legal-ytributario/certificaciones-pymes

Haggar, J., Soto, G., Casanoves, F., \& De Melo, E. (2017). Environmental-economic benefits and trade-offs on sustainably certified coffee farms. Ecological Indicators, 79, 330-337. doi:https://doi.org/10.1016/j.ecolind.2017.04.023

Heiss, G. (2017). Influencing factors and the effect of organizational capabilities on internationalization strategies for German SMEs in the MedTech Industry. Management Studies, 5(4), 263-277. doi:10.17265/2328-2185/2017.04.001

Huerta, M., Sandoval, S. A., \& Preciado, J. M. (enero-diciembre de 2016). Sistemas de calidad y desempeño empresarial: estudio de caso de empresas cárnicas en una región del noroeste de México. Ingeniería Industrial(34), 97-117.

Ibarra, M. A., González, L. A., \& Demuner, M. (2017). Competitividad empresarial de las pequeñas y medianas empresas manufactureras de Baja California. Estudios fronterizos, 18(35), 107-130. doi:http://dx.doi.org/10.21670/ref.2017.35.a06

Jadi, A. (2016). Enhancing the monotoring system of sfda in Saudi markets. International Journal of Software Engineering \& Applications, 7(4), 31-45. doi:10.5121/ijsea.2016.7404

Khatri, S., Ranjan, P., \& Grote, U. (2016). Fair Trade Certification and Livelihoods: A panel data analysis of coffee-growing households in India. Agricultural and Resource Economics Review, 45(3), 436-458. doi:https://doi.org/10.1017/age.2016.3

Kohne, R. (2019). Would further Marine Stewardship Council Sustainable Certification Improve Supply Chain Profitability in Australian Fisheries? Australasian Agribusiness Review, 27(1), 1-23. doi:10.22004/ag.econ.292471

Krishnan, S., Omar, C. C., Zahran, I., Syazwan, N., \& Alyaa, S. (2017). The awareness of gen Z's toward Halal food industry. Management, 7(1), 44-47. doi:10.5923/j.mm.20170701.06

Lajus, D., Stogova, D., \& Keskitalo, C. (2018). The implementation of Marine Stewardship Council (MSC) certification in Russia: Achievements and considerations. Marine Policy, 90, 105-114. doi:https://doi.org/10.1016/j.marpol.2018.01.001

Larios-Gómez, E., Cuevas-Vargas, H., \& Estrada-Rodríguez, S. (2016). El sistema de información de marketing (SIM) como estrategia competitiva de comunicación en las mipymes de México: estudio diagnóstico del impacto de las tic. Poliantea, 12(23), 129-163.

Latynskiy, E., \& Berger, T. (2017). Assessing the Income Effects of Group Certification for Smallholder Coffee Farmers: Agent-based Simulation in Uganda. Journal of Agricultural Economic, 68(3), 727-748. doi:https://doi.org/10.1111/1477-9552.12212 
Liñán , Á. (2017). Aspectos controvertidos de la libertad religiosa en España y Europa: Alimentación Halal y Casher. Estudios Constitucionales(2), 331-364. doi:http://dx.doi.org/10.4067/S0718-52002017000200331

Llamas, B., Del Carmen, R., \& Moreno, L. (2018). Impacto de la confianza en la percepción del consumidor. Revista De Investigación Académica Sin Frontera: División De Ciencias Económicas Y Sociales, 6(17).

López, G. (2019). Factores que influyen en la compra de alimentos orgánico en México. Un análisis mixto. Small Business International Review, 3(2), 69-85. doi:10.26784/SBIR.V3I2.210

Mehady, I., \& Rumana, Y. (2017). Impact of aquaculture and contemporary environmental issues in Bangladesh. International Journal of Fisheries and Aquatic Studies, 5(4), 100-107.

Méndez, J. (2017). Fusiones horizontales de empresas y estrategias de diferenciación de producto. Estudios de Economía, 44(2), 173-184. doi:http://dx.doi.org/10.4067/S071852862017000200173

Naranjo, R., Mercado-Caruso, N., Naranjo Oscar, \& Giraldo, M. (2018). Responsabilidad Social Empresarial (RSE) en medianas empresas del departamento del Atlántico, Colombia. Revista Venezolana de Gerencia, 23(84), 1-18.

Navarro, C., Pérez, J., \& Estrada, J. (2016). Guía de implementación del sistema integrado de gestión ISO 9001:2008 - ISO 22000:2005, para empresas de producción de leche entera pasteurizada y queso fresco. Revista Ingeniería Industrial UPB, 4(4), 103-108.

Ohmae, K. (1989). La mente estratega. Madrid: McGraw Hill.

Olsen, P., DeCarlo, A., \& Passarelli, S. (2017). WWJD? Frankincense distiller considers Fair Trade certification. Journal of Case Studies, 35(1), 102-113.

Procolombia. (12 de junio de 2018). Guía práctica - certificación HACCP (APPCC). Obtenido de Procolombia: http://www.procolombia.co/publicaciones/guia-practica-cetificacion-haccpappcc

Procolombia. (09 de julio de 2018). Guía práctica para conocer las certificaciones que se requieren para exportación de agroalimentos. Obtenido de Procolombia: http://www.procolombia.co/publicaciones/guia-practica-para-conocer-las-certificacionesque-se-requieren-para-exportacion-de-agroalimentos

Proecuador. (25 de junio de 2013). Guía de certificaciones internacionales. Obtenido de Proecuador: https://issuu.com/pro-ecuador/docs/guiacertificacionesh

Quintero, L. (2018). Estrategias de las multilatinas colombianas y su proceso de internacionalización. Revista Espacios, 39(42), 1-6.

Rangel, J., Arredondo, M. C., \& Espejel, I. (2017). ¿Estamos investigando la efectividad de las certificaciones ambientales para lograr la sustentabilidad acuícola? Sociedad y ambiente(15), 7-37.

Riaño-Casallas, M., Hoyos, E., \& Pacheco, I. (2016). Evolución de un sistema de gestión de seguridad y salud en el trabajo e impacto en la accidentalidad laboral: Estudio de caso en empresas del sector petroquímico en Colombia. Ciencia y Trabajo, 18(55), 68-72. doi:http://dx.doi.org/10.4067/S0718-24492016000100011

Rizo-Mustelier, M., Vuelta-Lorenzo, D. R., \& Lorenzo-García, A. M. (abril-junio de 2017). Agricultura, desarrollo sostenible, medioambiente, saber campesino y universidad. Ciencia en su PC(2), 106-120.

Soley, G., Wuyang, H., \& Vassalos, M. (2019). Willingness to Pay for Shrimp with Homegrown by Heroes, Community-Supported Fishery, Best Aquaculture Practices, or Local 
Attributes. Journal of Agricultural and Applied Economics, 51(4), 606-621. doi:https://doi.org/10.1017/aae.2019.19

Staricco, J., \& Naranjo, M. (2017). ¿Llevando justicia al comercio internacional? Una introducción a la propuesta de FairTrade. Trabajo y sociedad(30), 175-195.

Vargas, J., \& López, L. (2018). Efectos de la implementación de estrategias empresariales en los micronegocios. Neumann Business Review, 4(1), 44-56. doi:https://doi.org/10.22451/3002.nbr2018.vol4.1.10019

Vásquez-Tejos, J., \& Torres-Vallejos, J. (2018). Caracterización de los auditores de sistemas de gestión pertenecientes a empresas certificadoras en Chile. Signos, 10(2), 55-69. doi:10.15332/s2145-1389.2018.0002.03

Veliz, A., \& Díaz, W. (2019). Manual de procesos aplicado a la certificación del sistema de gestión en control y seguridad BASC. Revista Observatorio de la Economía Latinoamericana(5), $1-11$.

Vera, J. A., \& Cañón, J. E. (2018). El valor agregado de un sistema de gestión ambiental más allá de la certificación. Revista de la Facultad de Ciencias Básicas, 16(1), 86-91. doi:10.24054/01204211.v1.n1.2018.2929

Villa, M. (2016). From Multilatina to Global Latina: Unveiling the corporate-level international strategy choices of Grupo Nutresa. AD-minister(29), 23-57. doi:10.17230/ad-minister.29.2

Wibowo, M. W., \& Ahmad, F. S. (2016). Non-muslim consumers' Halal food product acceptance codel. Procedia Economics and Finance, 37, 276-283. doi:https://doi.org/10.1016/S22125671(16)30125-3

Wijen, F., \& Chiroleu-Assouline, M. (2019). Controversy over voluntary environmental standards: A socioeconomic analysis of the Marine Stewardship Council. Organization \& Environment, 32(2), 98-124. doi:https://doi.org/10.1177/1086026619831449

Zhang, J., \& Boldt, L. (2017). Is organic labelling enough? Information disclosure as policy instrument to empower consumer choices. Hawaii International Conference on System Sciences, 2701-2710. doi:10.24251/HICSS.2017.326 